\title{
MEMBRANAS E ANÉIS ESOFÁGICOS
}

\author{
Esophageal membranes and rings \\ Erickson José Blum LIMA, Daniélle Toniolo MALAFAIA, Sebastião Gonzaga BARBOSA-NETO, \\ Jair TABCHOURY-FILHO, Fábio Roberto Ruiz de MORAES, \\ Gilmar Pereira SILVA, Mery Tossa NAKAMURA
}

ABCDDV/558

Lima EJB, Malafaia DT, Barbosa-Neto SG, Tabchouy-Filho J, Moraes FRR, Silva GP, Nakamura MT.Membranas e anéis esofágicos. ABCD Arq Bras Cir Dig 2007; 20(3):201-4.

RESUMO - Racional - As membranas e anéis esofágicos são entidades pouco freqüentes e, por isso, fica difícil o aprofundamento de seus aspectos clínicos e diagnósticos, sendo assim oportuna revisão do tema. Método - Foi realizada revisão da literatura internacional através do Pubmed (www. pubmed.com) e nacional (www.lilacs.br) utilizando-se as seguintes palavras-chave: esôfago, membranas, etiologia, diagnóstico. A extensão do tema foi limitada aos seguintes enfoques: conceitos, etiologia, epidemiologia, etiopatogenia e diagnóstico. Conclusão - Embora a literatura apresente bom entendimento do desenvolvimento dessas afeç̧ões, estudos devem ser continuados para aprofundar os conhecimentos existentes e melhor orientar a conduta a ser tomada nos seus portadores.

DESCRITORES - Esôfago. Membranas. Etiologia. Diagnóstico.

\section{INTRODUÇÃO}

As membranas e anéis esofágicos são entidades pouco freqüentes e, por isso, fica difícil o aprofundamento de seus aspectos clínicos e diagnósticos. De um modo geral, a equipe de saúde que atende os pacientes deles portadores ficam indecisos com a conduta a ser tomada. Assim, revisão e atualização do tema é oportuna.

\section{MÉTODO}

Foi realizada revisão da literatura internacional através do Pubmed (www.pubmed.com) e nacional (www.lilacs. br) utilizando-se as seguintes palavras-chave: esôfago, membranas, etiologia, diagnóstico. A extensão do tema foi limitada aos seguintes enfoques: conceitos, etiologia, epidemiologia, etiopatogenia e diagnóstico.

\section{REVISÃO DA LITERATURA}

\section{Membranas}

\section{Conceito}

Membrana esofágica é definida como estrutura fina que consiste de pregas de mucosa que se projetam parcial ou totalmente no lúmen esofágico.

Pode ser de origem congênita ou adquirida e localizar-

Trabalho realizado no Hospital Brasília, Brasília, DF, Brasil

Endereço para correspondência: Erickson José Blum Lima, e-mail: erickson@hobra.com.br se em qualquer lugar do esôfago. Normalmente é recoberta por epitélio escamoso. Geralmente, a membrana é única, mas pode haver duplas ou múltiplas.

\section{Epidemiologia}

As mais comuns são localizadas no esôfago cervical e fazem parte de síndrome inicialmente descrita por Paterson $^{1}$ e Kelly ${ }^{2}$ em 1919 e, posteriormente, por Vinson ${ }^{3}$ em 1992. São mais freqüentes no sexo feminino ( $80 \%$ a $90 \%$ ), entre 40 e 70 anos de idade, sendo incomum antes dos 30 anos de idade.

Caracteriza-se por associar-se à anemia ferropriva, queilite angular, pele seca, glossite, onicodistrofia (Figura 1 ), perda de peso e disfagia. Ocorre mais freqüentemente entre europeus (norte da Europa), especialmente nas áreas rurais da Suécia ${ }^{4}$.

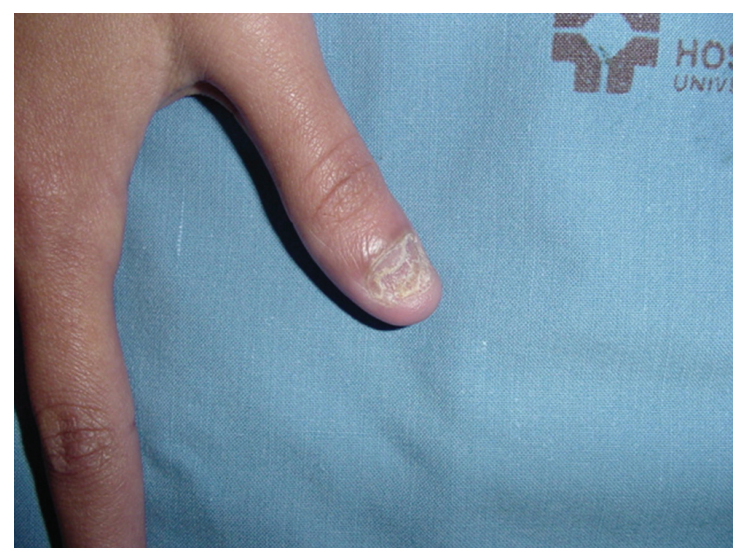

FIGURA 1 - Paciente mostrando associação com onicodistrofia 
As membranas do esôfago superior incidem em cerca de $5 \%$ a $15 \%$ dos pacientes com disfagias e podem ser achado endoscópico em até 1,3\% de pacientes assintomáticos.

\section{Etiopatogenia}

A etiopatogenia da síndrome e das membranas é desconhecida, tendo sido propostos vários mecanismos como fatores genéticos, nutricionais, auto-imunes e infecciosos. Alguns autores ${ }^{5,67,8}$ acreditam que a coexistência das membranas e da anemia poderia ser apenas casual, posto que muitos com membrana não apresentam anemia. Contudo, o papel preciso da deficiência de ferro no desenvolvimento da síndrome de Plummer-Vinson ainda está por ser definida.

A disfagia parece relacionada não apenas pela presença de membrana esofágica, mas também por contração muscular inadequada devido à deficiência de ferro. Isso levaria à redução da propulsão do bolo alimentar pelo músculo cricofaríngeo e, segundo hipóteses recentes, incoordenação da musculatura lisa esofágica ${ }^{9}$.

Segundo observações de alguns autores na literatura, ${ }^{6,8}$, ao analisarem videofluoroscopias, existe na região esofágica cervical deficiência de dilatação da parede anterior ao deglutir o bário. Com alimentos sólidos, essa dilatação é passiva e não há formação de membranas no esofagograma e esofagoscopia. Portanto, concluem que as membranas podem ser causadas por restrição da dilatação das paredes esofágicas ao deglutir líquidos, o que resultaria de inflamações repetitivas com processo de cicatrização subseqüente.

\section{Diagnóstico}

Além dos achados de exame clínico, característicos da síndrome de Plummer-Vinson e a associação com a deficiência de ferro, outros exames auxiliam no seu diagnóstico.

O estudo contrastado do esôfago nem sempre demonstra a membrana esofágica, podendo, muitas vezes, passar despercebida no primeiro exame. Novo exame, realizado com projeção lateral, tende a visualizar melhor o anel (Figura 2), pois em alguns casos a projeção ânteroposterior isoladamente pode não demonstrar a membrana esofágica ${ }^{10}$.
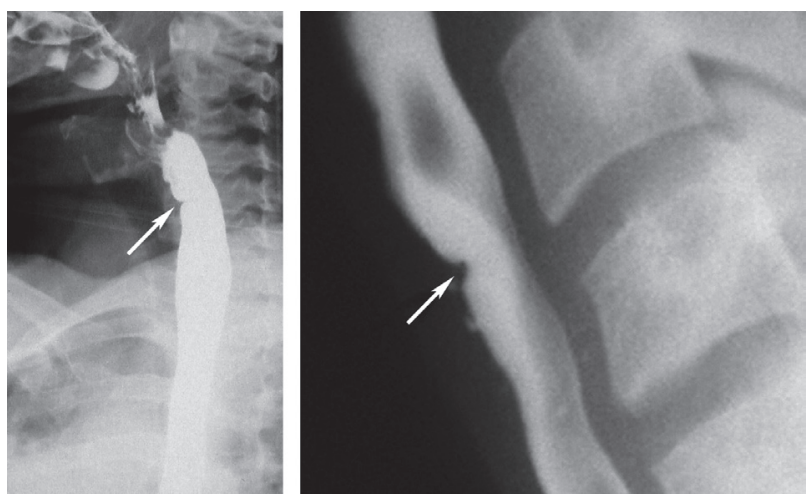

FIGURA 2 - Estudo radiográfico contrastado de dois casos de membrana esofágica
O videodeglutograma pode apresentar melhor sensibilidade que o exame contrastado, por ser estudo dinâmico. A endoscopia geralmente faz o diagnóstico na totalidade dos casos. A membrana tem aparência acinzentada, fina, com pequenas arteríolas e vênulas e com lúmen centralizado ou lateralizado. Algumas vezes, o anel é tão fino que pode ser rompido facilmente com a ponta do endoscópio. Outras vezes, a membrana pode apresentar-se elástica e complacente.

A avaliação manométrica da motilidade esofágica pode evidenciar aumento relativo da amplitude das ondas peristálticas, porém o padrão não caracteriza esôfago em quebra-nozes ${ }^{8,11,12}$.

\section{Anel Esofágico}

\section{Conceito}

Anel esofágico, também chamado anel de Schatzki ${ }^{13}$, foi descrito inicialmente em 1953 por Ingelfinger, Kramer e Schatzki ${ }^{12}$. Consiste em uma subestenose localizada na transição anatômica esofagogástrica no ponto de união do epitélio escamocolunar.

\section{Etiopatogenia}

Encontra-se com maior freqüência em adultos e idosos, o que não afasta a possibilidade de ser diagnosticado em crianças ou de ser congênito, com sintomatologia tardia ${ }^{12,14}$. A etiologia do anel de Schatzki não está totalmente esclarecida. É difícil determiná-la, porque, não havendo refluxo clinicamente evidente e ocorrendo mais freqüentemente em jovens, pode-se inclusive aceitar a possibilidade de etiologia congênita. Sabe-se que, em quase sua totalidade, é enfermidade adquirida, tendo como causa a agressão repetida do epitélio esofágico por refluxo (Figura 3).

O refluxo gastroesofágico ocasiona lesões esofágicas que, inicialmente espásticas, evoluem para fase inflamatória e posteriormente para fibrose. Pode ter características próprias, produzindo estenose de extensão e gravidade variada na dependência de múltiplos fatores, sendo os principais o tempo de exposição ao refluxo, integridade do clareamento esofágico e o $\mathrm{pH}$ do líquido refluído.

Sua freqüência é alta e estima-se que pode ser encontrado entre $15 \%$ a $18 \%$ de pacientes adultos submetidos a exames gastrointestinais de rotina.

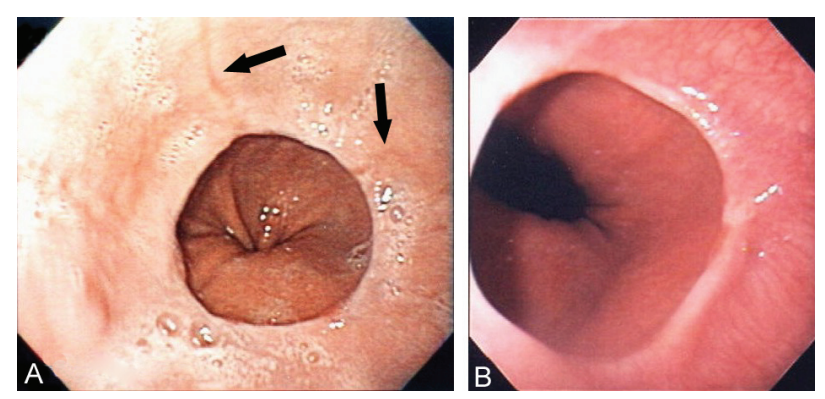

FIGURA 3 - Estudo endoscópico mostrando anel de Schatzki: em A com esofagite (seta) e em B sem esofagite 


\section{Anatomia patológica}

O revestimento epitelial do anel esofágico apresenta, na sua parte superior, epitélio escamoso estratificado e na inferior, epitélio colunar do tipo gástrico. O núcleo central é composto por tecido conjuntivo vascularizado freqüentemente contendo células inflamatórias. Tem espessura de 2 a $4 \mathrm{~mm}$ e raramente faz protrusão no lúmen do esôfago. $\mathrm{O}$ aspecto é de tecido fibrótico com vários graus de estenose e está restrito à submucosa. No esôfago aberto ele se apresenta como pequena elevação lisa.

\section{Quadro clínico}

Assim como em outras numerosas doenças esofágicas, a disfagia é o principal sintoma ${ }^{14,15}$, com característica de ser intermitente para sólidos ${ }^{16,17,18}$ e líquidos e evolução progressiva com intervalo de vários anos, tornando-se, assim, crônica. Outra característica dela é sua sensação que ocorre em qualquer segmento do esôfago torácico, mesmo sendo o anel de localização baixa.

Queimação retroesternal (pirose) é sintoma freqüente em pacientes portadores de anel esofágico em razão da associação com a doença do refluxo gastroesofágico. Outra manifestação é a impactação de alimentos sólidos no local do anel, principalmente em homens idosos. Alguns pacientes podem referir regurgitação e broncoaspiração associados à disfagia.

\section{Diagnóstico}

O exame que pode fornecer mais detalhes do anel esofágico é a fluoroscopia esofágica. Mesmo não sendo o primeiro exame a ser solicitado, deve ser realizada quando a história clínica sugere e a endoscopia digestiva alta é normal. Smith et al. ${ }^{19}$ referem incidência de $20 \%$ de anel em pacientes com disfagia, quando investigado com exame contrastado do esôfago (Figura 4). Por meio da videofluoroscopia e deglutograma estuda-se todo o esôfago durante a deglutição, simula-se a ingestão de alimentos líquidos pastosos e sólidos e visualiza-se o ponto de estenose. É excelente meio de visualização do anel e pode também definir o seu diâmetro, determinação importante na escolha do melhor método de tratamento.

A endoscopia digestiva alta deve ser solicitada, principalmente, para excluir causa maligna da disfagia. Para fazer-se o diagnóstico por endoscopia digestiva, o médico deve ser experiente e ter informações sobre a clínica apresentada, pois as alterações endoscópicas são discretas e podem passar despercebidas (Figura 3B).
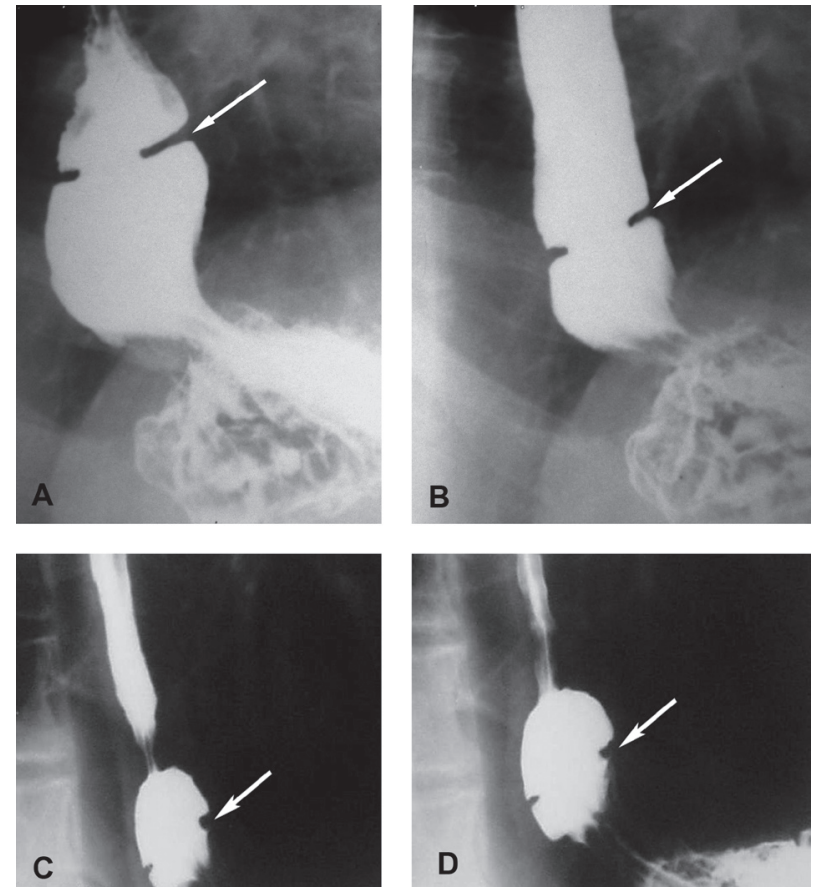

FIGURA 4 - Estudo radiográfico contrastado mostrando nas setas quatro casos de anel de Schatzki

O estudo da motilidade esofágica por intermédio da manometria também auxilia no diagnóstico evidenciando contrações com amplitude alta, longa duração e múltiplos picos de contração, com esfíncter esofágico inferior normal.

No diagnóstico diferencial, devem-se excluir as lesões esofágicas extrínsecas, estenose péptica, distúrbios motores e carcinoma de esôfago, os quais podem ser facilmente identificados com os exames anteriores ${ }^{20,21,22}$.

\section{Prognóstico}

A evolução clínica dos pacientes não tratados é progressivamente disfágica, podendo ser estacionária em graus de constrição variáveis. Dependendo do diâmetro no qual a evolução se delimita, a clínica será mais ou menos evidente. Contudo, em função do processo inflamatório da doença do refluxo gastroesofágico associado na quase totalidade dos casos, a pirose pode ser intensa, chamando mais atenção ao processo de esofagite do que o próprio anel.

Quando se trata a doença do refluxo, o quadro clínico se estabiliza e muito poucas vezes há necessidade de se atuar sobre o anel. Quando tal necessidade se faz presente, a dilatação endoscópica pré-operatória quase sempre é suficiente para se obter qualidade de vida permanente boa.

Lima EJB, Malafaia DT, Barbosa-Neto SG, Tabchouy-Filho J, Moraes FRR, Silva GP, Nakamura MT. Esophageal membranes and rings. ABCD Arq Bras Cir Dig 2007; 20(3):201-4.

ABSTRACT - Background - Esophageal membranes and rings are uncommon entities, being difficult to study and deepen clinical and diagnostic knowledge pertaining these structures, making this article an opportune review. Methods - A literature review was made using Pubmed (www. pubmed.com), being this one an international one, and a national review was made utilizing (www.lilacs.br) the following key-words: esophagus, membranes, etiology, diagnosis. The theme was limited to the following: concepts, etiology, epidemiology, etiopathogeny and diagnosis. Conclusion - Eventhough literature presents a good understanding of the development of these affections, further study and research must be continued in order to deepen the knowledge already known, as well as to better orientate the conduct taken pertaining patients who have it.

HEADINGS - Esophagus. Membranes. Etiology. Diagnosis. 


\section{REFERÊNCIAS}

1. Barthlen W, Feussner H, Hanning C, et al. Surgical therapy of Zenker's diverticulum: low risk ang high efficiency. Dysphagia. 1990;5:13-9.

2. Coelho JC. Aparelho digestivo: clínica e cirúrgica. São Paulo: Medsi; 2004. p.119-21

3. Devault KR. Lower esophageal (Schatzki's) ring: pathogenesis, diagnosis and therapy. Dig Dis Sci. 1996;14:323-9.

4. Disario JA, Pedersen PJ, Bichis-Canoutas C, et al. Incision of recurrent distal esophageal (Schatzki) ring after dilation. Gastrointest Endosc. 2002;56:2448.

5. Gawrieh S, Carroll T, Hogan WJ, et al. Swallow syncope in association with Schatzki ring and hypertensive esophageal peristalsis: report of three cases and review of the literature. Dysphagia. 2005;20:273-7.

6. Hirano I, Gilliam J, Goyal RK. Clinical and manometric features of the lower esophageal muscular ring. Am J Gastroenterol. 2000;95:43-9.

7. Inque M, Nakanishi R, Osakit T, et al. Esophagopleural fistula originating from diverticulum after pneumonectomy. A case report and review of the literature. J Cardiovasc Surg. 1999;40:761-3.

8. Kelly AB. Spasms at the entrance of the esophagus. J Laringol Rhinol Otol. 1919;34,285-9.

9. Malafaia O, Moraes RS, Baretta GAP. Membrana esofágica cervical (síndrome de Plummer-Vinson): remissão completa com reposição de ferro via oral. ABCD Arq Bras Cir Dig. 2004;17:65-6.

10. Morrow JB, Vargo JJ, Goldblum JR, et al. The ringed esophagus: histological features of GERD. Am J Gastroenterol. 2001;96:984-9.

11. Novacek G. Plummer-Vinson syndrome. Orphanet J Rare Dis. 2006;1:36.

12. Ott DJ, Kelley TF, Chen MY, et al. Use of a marshmallow bolus for evaluating lower esophageal mucosal rings. Am J Gastroenterol. 1991;86:817-20.
13. Paterson DR. A clinical type of dysphagia. J Laryngol Rhinol Otol. 1919;34:289-91.

14. Pereira JC, Marconcini JF, Marconato MC, et al. Síndrome de Plummer-Vinson: relato de caso e revisão da literatura. J Bras Med. 2000;79:46-51.

15. Petroianu A. Terapêutica cirúrgica. Rio de Janeiro: Guanabara Koogan; 2001.

16. Rohl L, Aksglaede K, Funch-Jensen P, et al. Esophageal rings and strictures. Manometric characteristics in patients with food impaction. Acta Radiol. 2000;41:275-9.

17. Schatzki R, Gary JE. Dysphagia due to a diaphragm-like localized narrowing in the lower esophagus ('lower esophageal ring'). Am J Radiol. 1953;70:911.

18. Scolapio JS, Pasha TM, Gostout CJ, et al. A randomized prospective study comparing rigid to balloon dilators for benign esophageal strictures and rings. Gastrointest Endosc. 1999;50:13-7.

19. Sgouros SN, Vlachogiannakos J, Karamanolis G, et al. Long-term acid suppressive therapy may prevent the relapse of lower esophageal (Schatzki's) rings: a prospective, randomized, placebo-controlled study. Am J Gastroenterol. 2005;100:1929-34.

20. Smith DF, Ott DJ, Gelfand DW, et al. Lower esophageal mucosal ring: correlation of referred symptoms with radiographic findings using a marshmallow bolus. AJR Am J Roentgenol. 1998;171:1361-5.

21. Uygur BO, Tuncer K, Dolaioglu C. Plummer Vinson syndrome presenting with an esophageal stricture. J Clin Gastroenterol. 1999;29:291-2.

22. Vinson PP. Hysterical dysphagia. Minn Med. 1922;5:107-8.

Conflito de interesse: não há Fonte financiadora: não há Recebido para publicação em: 04/04/2007 Aceito para publicação em: 15/05/2007 